\title{
PERSONAL REFLECTIONS ON A TALE OF TWO BOOKS: SOCIAL AND ENVIRONMENTAL ACCOUNTING RESEARCH IN THE PAST, PRESENT AND FUTURE
}

\author{
Reg Mathews \\ Charles Sturt University
}

\section{BACKGROUND/PREFACE}

I was greatly honoured and not a little surprised to be asked to participate in this venture. Of course, I was relieved that the editors (Gray and Guthrie, 2007) (hereinafter GG 2007) took the European view of the Festschrift as a celebration of an event (my retirement), rather than the Australasian view (after the demise of the person concerned).

Now that the work has been published (GG 2007), I have had the opportunity to study the contributions of my contemporaries as well as seeing some of my own work in print once again. Together the contributions mark both past and present rather than the future of Social and Environmental Accounting Research (SEAR), which is increasingly being called Sustainability Accounting (SA).

\section{INTRODUCTION}

This paper was requested by the guest editors of the Australasian Accounting, Business and Finance Journal (AABFJ) following the Sydney CSEAR conference. One topic suggested to me was to write a comment on recent developments leveraging on GG 2007 or that part that has not been published previously. I am pleased to attempt this but wish also to make reference to another book published at the same time (Unerman, Bebbington and O'Dwyer (UBOD 2007), because I believe that the different perspectives presented are informative for all scholars in the field of SEAR which is now often referred to as SA.

The remainder of this paper other than the introduction and conclusion will consist of firstly a review of GG 2007, secondly a review of UBOD 2007, and thirdly a number of observations based on these contrasting works.

\section{A REVIEW OF GG 2007}

GG 2007 is made up of two parts. The first part consists of an editorial and ten contributions by a total of thirteen academics currently working in Australia, Canada, New Zealand, the United Kingdom, and the United States. The second part consists of reprints of nine papers by Mathews, three of which are joint publications with those represented in the collection of editorial and ten contributions. There is also a detailed bibliography of works by Mathews ranging from 1984 to 2004.

The overall characteristic of the nine papers is that with the exception of the papers by Milne and Reynolds, they are concerned with Social and Environmental Accounting with no reference to sustainable development, sustainability accounting, eco-justice or any of the language more recently introduced to the field. A second characteristic is that whereas the 
acceptance of a critical theorist stance is perceived as very important to sustainability accounting (Dillard, UBOD 2007, p.37), the social and environmental accounting writers have no one philosophical base.

The topics covered the following areas: Deegan (Chapter 2; Social Accounting Research: An Australasian Perspective) attempts to answer a series of research questions about the extent to which Australasian based academics have been involved in social accounting research, the level of concentration, the focus of their work, and the journals employed as outlets for this research. The results were encouraging. Using a sample of nine internationally rated journals, it was found that within social accounting Australasian academics are 'punching above their weight'. However, the number of researchers was not large and:

This is of concern as it would be preferable, perhaps, to see more voices in the debate- rather than the same voices promoting the same opinions (GG 2007, p.16).

Gordon (Chapter 3; The Challenge of Social Accounting) takes a North American perspective in looking at research/publication in social accounting. Research using two common North American databases (ABI/Inform and Business Source Premier) found details of social accounting articles published in a range of journals. It is no surprise to find that the majority of these journals are British Commonwealth based (AAAJ, AF, AOS, BAR, CPA). The only North American based journals involved are JAPP and JBE. This bias is confirmed by a geographical analysis based on North America versus the rest of the world.

After the usual complaints of North American academics that tenure and promotion decisions are all too often based on publication in the area of financial markets, Gordon recommends that the way forward for social accounting is through the development of additional high level specialist journals, battling mainstream economics based researchers in their own field to give the 'creed of greed' a conscience, and to show that the most interesting problems cannot be solved by recourse to mathematics.

Lehman (Chapter 4; Ethics, Communitarianism and Social Accounting) attempts to combine his research interest, which is a branch of philosophy, with social accounting. Lehman credits social accounting with enhancing the interest of accountants and the profession in questions of ethics, but notes that these developments have been slow in developing and may lead to a loss of some aspects of the discipline to the law, or to overseas competitors.

Lockhart (Chapter 5; A festive Trip-le Step Across the Dance Floor of Environmental Accounting Education), details aspects of the authors life as a radical-humanist educator and a course of study on Environmental Accounting. Lockhart indicates that some of the problems with the course may be because of the 'banking' nature of the programme, which will be amended to reflect a 'dialogic' approach as advocated by Bebbington and Thomson (2001). This will assist the students in acquiring the skills needed to "...contribute substantively to the environmental debate in their role as professionals” (Lockhart, GG 2007, p.48).

Milne (Chapter 6; Downsizing Reg (Me and You)! Addressing the 'real' Sustainability agenda at Work and Home). Milne begins this paper with a review of Mathews (1984) and examines each of the classifications used in that paper. The next section is an attempt to link 
micro and macro indicators using the Landcare Annual Carbon Impacts as an example. The final section contains a novel account of the need for all of us to consider downsizing, and this is exemplified by the cost to the environment of air travel for a successful academic. This is summarized as follows:

Being able to make the necessary connections between our own individual and household (and for some of us our workplace) activities, such that we take ecologically informed choices and develop ecologically benign routines and habits, is critical, but not something that environmental accounting research and practice seems well placed to address (GG 2007, p.60).

This use of social accounting in the context of the family/individual bears some resemblance to the work of the late Trevor Gambling (Gambling, 1974).

It should be noted that Milne is the only author (in GG 2007) to refer to sustainability or to use the language used in UBOD 2007.

Owen (Chapter 7; Social and Environmental Accounting: Celebrating a Silver Jubilee of Engagement and Community) builds on a suggestion that social and environmental accounting must lead to changes in the relationship between business, the stakeholders and the environment. This interaction has become known as engagement and community. Owen subdivides the topic for discussion into detached engagement, practical engagement, and engagement with nonmanagerial interests, and a note on community. He concludes that:

Whilst the academic contribution of SEA engagement based research therefore is clearly discernible, considerable doubt must be expressed as to its practical impact. Indeed how could this be otherwise in an increasingly rapacious business climate where, despite the lip service paid to notions of social responsibility, the sole aim is to maximize shareholder wealth whatever the social and environmental cost? Would it be better to abandon the attempt and, in common cause with the critical theorists seek to become an integral part of alternative social movements? (GG 2007, p.73).

Parker (Chapter 8; The Editorial Board Role in Journal and Scholarship Development) describes the roles of editors and editorial board members in the selection, review, and publication of academic papers and the encouraging of scholarship within a given discipline. The role of the editorial board member goes beyond that of reviewer and may involve adjudication and even have an impact on policy.

Perera (Chapter 9; The International and Social aspects of Social Accounting) considers the relationship of culture and social accounting under several headings including international aspects of CSR, regulating CSR, legitimacy theory and CSR, and the cultural relativity of CSR. The latter has an important impact on research:

For example, hypotheses are tested using observable evidence, gathered using particular techniques which are amenable to statistical analysis. If the evidence is not observable, then the hypothesis is rejected as not being supported. But absence of evidence does not always mean evidence of absence. Evidence may not be there to observe in some cases due to cultural reasons, such as the influence of religion (GG 2007, pp.9697).

Generalisability may also be affected by culture. 
Reynolds (Chapter 10; Accounting, Communication, Social Responsibility and Justice-A Short Essay) has written a philosophical paper in which she asks To whom is responsibility owed? How is communication determined? How is social responsibility measured and reported? And is social justice a possible outcome? Reynolds concludes:

This time is a critical time for the accountant as ethical actor to elaborate and acknowledge both the power and the responsibility in the functions they have undertaken for society. Reports by their nature are not value neutral so there is increased obligation for the accountant to understand his/her own ontological, ecological and ethical framework in order to serve effectively in the communications and therefore the decisions of an emergent society (GG 2007, p.104).

Tozer and Hamilton (Chapter 11; Re-energising the Social Contract in Accounting: The Case of James Hardie Industries) recall the long and disgraceful tale of attempts to avoid legal and moral responsibility for asbestos-related health problems suffered by employees of James Hardie and related companies. The basic issues explored are the violation of a social contract and lack of adequate legal protection for claimants when the corporation de-listed in Australia and relisted in The Netherlands, together with the eventual acceptance of the need to provide more adequate funding for affected claimants which was brought about by public pressure.

\section{UBOD 2007}

This volume of edited papers appeared at the same time as the Festschrift (GG 2007) and in many respects is a related volume; it is the same size, almost the same coloured cover, and four of the contributors are common to both works (Deegan, Owen, Milne and Gray). However, in almost all other respects they are quite different books. UBOD 2007 is about sustainability accounting and accountability and looks to the future not the past or the present. My interest in this book stems from being asked to review it for the British Accounting Review (BAR). In the course of preparing a review I was struck by the difference between GG 2007 which is very much in the 'Mathews style' even though excellently written by my peers (that is, it is limited to social and environmental accounting and mostly is not influenced by critical theory) and UBOD 2007, this more powerful account of 'things as they ought to be'. When reading both books I had a feeling of being at a point almost of discontinuity in the field which is why the approach of this paper is to review each, and attempt to identify the major differences in toto as well as through individual contributions.

In the course of writing my BAR review I actually prepared two. One was much too long for BAR and will be enlarged further and used as the basis for the remainder of this section.

This is a book of readings edited by three well-known researchers in the field. The edited book format is an unusual format for this field, the last comparable work that comes to mind is that of Owen (1992). The editors have compiled 18 chapters within four logical groupings. The editors themselves have contributed three individual papers and one jointly authored paper, with the remaining 14 papers contributed by a total of 19 authors from nine countries. Readers are therefore exposed to the thoughts and scholarship of a total of 22 individuals, all noted for their contributions to this field. For students and beginning researchers this is an important aspect of the publication, and this feature is reinforced by the detailed list of contributors provided. 
The Foreword is by Jonathon Porritt who defines sustainable development in the following terms:

Sustainable development is the central public policy goal of our times. It is the only 'big idea' that provides the moral basis for grappling with the twin challenges of achieving ecological and social sustainability. The United Kingdom recognizes two overarching priorities for sustainable development: living within environmental limits, and ensuring a strong, healthy and just society (UBOD 2007, p.xvii).

The three editors are responsible for an introductory chapter which sets the scene for the whole book, and outlines the content of each chapter; and also for a concluding chapter at the end of the book. The editors 'nail their colours to the mast' with a statement that resembles that of Porritt in the Foreword:

Many people argue that the growing social injustice experienced by ever larger numbers of people, and the growing damage to the ecosphere, are a result of a dominant - and almost unquestioned- objective of maximizing economic growth. In these terms (and several arguments supporting this linkage are discussed in a number of papers in this book), economic growth (characterized by energy and material-intensive production and exploitative social relations) is socially and environmentally unsustainable (UBOD 2007, p.2).

Section I is entitled; Setting the context for sustainability accounting and accountability and contains two chapters. Chapter 1 (Thomson; Mapping the terrain of sustainability accounting) uses eleven dimensions ranging from conceptual maps to the evaluatory frameworks used, research methods and research topics. The author presents a comprehensive treatment of a diverse literature, before concluding that:

The intention of these alternative representations of the sustainability accounting field is to challenge and problematise others' preconceptions of the literature and inform the ongoing development of sustainability accounting (UBOD 2007, p.35).

Clearly this intention is now likely to be realized as beginning researchers consider the framework offered.

Chapter 2 (Dillard; Legitimating the social accounting project: an ethic of accountability) is a philosophical paper in which the author surveys the bases for social accounting and accountability, concluding that a critical theory lens (Neomarxist social theory, Frankfurt School) is the most appropriate one to use:

Applying critical theory as an empowering philosophy for social accounting provides insights into its idealized objectives, a basis for designing research and application programmes as well as a set of criteria by which progress can be measured (UBOD 2007, p.40).

Dillard thus suggests that to be an advocate of social accounting one is also likely to be a critical theorist:

In summary, the energetic and pragmatic approach taken by the social accounting project is compatible with, and a consequence of, its acquaintance with critical theory (UBOD 2007, p.42).

After discussing the 'ethic of accountability', Dillard concludes with a message that: 
members of the accounting academy must recognise and embrace the scope of the social accounting project, ensuring that it is represented in the accounting curriculum and in their research programmes (UBOD 2007, p.49).

This is an interesting paper which appears to incorporate both traditional references to the social accounting project and to sustainability and sustainable accounting. Perhaps Dillard is able to operate in both the past/present and future domains. Some of the contributors to GG 2007 do not share the same enthusiasm for critical theory.

In section II, which deals with external reporting of sustainability policies and practices, Buhr (chapter 3) begins Histories of and rationales for sustainability reporting with a statement that she is not convinced that there is currently any sustainability reporting and goes on to suggest that this may be an unachievable ideal. The chapter details a brief history of sustainability reporting through social, environmental, and triple bottom line reporting showing that there are nearly insurmountable problems in achieving sustainable accounting reports. The chapter concludes that although sustainability reporting cannot save the planet by itself, to pursue this form of reporting may lead to desired changes in management strategies and information systems, which in turn may lead to less unsustainable behaviour by all parties.

In chapter 4 Adams and Narayanan consider the standardization of sustainability reporting. Both Buhr and Adams and Narayanan use the definition of sustainability from the Brundtland Report but they have very different approaches; Buhr as stated above, is somewhat concerned about the widespread use of the term SA where it does not exist, and doubts whether it can exist whereas Adams and Narayanan show no hesitation. They state:

The issue of sustainability is gaining prominence in the agenda of governmental and non-governmental organizations which are increasingly putting pressure on companies to incorporate sustainable practices into their business operations (Adams and Narayanan, UBOD 2007, p.70).

The majority of the chapter is devoted to a description of bodies that promote sustainability reporting including the Global Reporting Initiative (GRI), International Standards Organisation (ISO), the World Business Council for Sustainable Development (WBCSD), the Institute of Social and Ethical Accountability (AA1000), the Sustainability Integrated Guidelines for Management (SIGMA) project, the United Nations Global Compact and initiatives by Canadian and Australian organizations. A useful comparison is provided at page 81 of the guidance provided by various bodies. The work of the EU as well as Australia and Canada on developing conceptual frameworks for SA is clearly very important.

Although otherwise comprehensive, the chapter does not consider any of the normative disclosure models proposed by academics. Also, the importance of audit/assurance is also not considered in this chapter. Although a relatively optimistic paper overall, the authors conclude:

Despite evidence that corporate social responsibility pays off in bottom line benefits, without mandatory reporting guidelines focusing on processes of reporting and governance structures, some companies will continue to produce reports which leave out impacts which are material to key stakeholder groups (Adams and Narayanan, UBOD 2007, p.83). 
In Chapter 5, Unerman considers stakeholder engagement and dialogue. A variety of issues related to the role of stakeholder engagement and dialogue in the process of social and environmental reporting are considered. These practices are located in the context of the motives for engagement in social and environmental reporting. The author concludes that unless dialogue can be achieved between reporters and stakeholders the reporting that takes place will not assist in furthering sustainability. Specifically:

If we are to achieve an improvement in the sustainability of business, then stakeholder dialogue mechanisms which give greater empowerment to a broad range of stakeholders will need to be developed and employed. Otherwise stakeholder dialogue may continue to be used to provide a fig-leaf for strategically motivated social and environmental reporting which has little to do with making business more holistically sustainable in practice (UBOD 2007, pp.100-101).

Tilt (Chapter 6 External Stakeholders’ perspectives on sustainability reporting) begins by stating "Corporate Social Disclosure (CSD), more recently termed Triple Bottom Line or Sustainability Reporting ...” (Tilt, UBOD 2007, p.104) which is an interesting difference from those who appear to be implying that SA is a new topic with different characteristics. Tilt discusses the perspectives of external stakeholders, shareholders and investors, insurers and banks, consumers and suppliers, employers and trade unions, non-governmental organisations (NGOs), the media and the general public as reported in a wide range of studies. The ACCA reporting awards are examined in detail.

One researcher who has employed organisational legitimacy widely in his research and writing is Craig Deegan, who contributed Chapter 7 entitled Organisational Legitimacy as a motive for sustainable reporting. Deegan outlines organisational legitimacy theory and its relevance to the social contract, the legitimacy gap and organisational reaction, followed by legitimacy strategies and their limitations, concluding with some thoughts about the way forward for legitimacy theory as a research tool. For the purposes of this paper Deegan makes two very important statements. Firstly, "central to legitimacy theory is the theoretical construct known as the social contract" (UBOD 2007, p.127) and "legitimacy theory - which is embedded in the 'bourgeois' branch of political economy theory - does not consider or question structural or class-based conflicts within society” (UBOD 2007, p.131). Thus challenging the view put forward by Dillard regarding the dominance of the critical theoretical perspective in SA.

A complementary view is provided in Chapter 8 (Larringa-Gonzalez; Insights from Neoinstitutional Theory). The chapter provides an examination of neoinstitutional theory, a discussion of institutionalisation and its relationship to change and finally the relationship between neoinstitutionalism and legitimacy theory, thus relating chapters 7 and 8:

As regards the commonalities and differences between neoinstitutionalism and the often used legitimacy theory, it seems that the latter is a particular case of the former, related to the regulative pillar (LarringaGonzalez, UBOD 2007, p.164).

As noted above, the issue of assurance is not included in Chapter 4. This occurs in Owen (Chapter 9; Assurance practice in sustainability reporting) who addresses a crucial area in any reporting context. Recent trends are considered together with moves towards the standardization of practice. These sections are followed by a critical overview of current assurance practice, and finally a section on the future development of sustainability assurance practice. Owen reports some progress but at the same time is concerned at a lack of legislative backing: 
Essentially what is missing in the whole debate over the development of sustainability assurance is some intervention by regulatory authorities in the public policy domain, designed to bring about a greater level of corporate accountability to stakeholder groups (UBOD 2007, p.180).

The paper concludes that reliance on notions of stakeholder engagement, and current practices of sustainability assurance do not provide countervailing power to hold preparers to account.

Future prospects for corporate sustainability reporting (Milne and Gray, Chapter 10), is the final paper in Part II. The authors review the recent use of sustainability reports by large companies on a global basis including, including stand alone reports. The quality of reports is considered using the ACCA sustainability report criteria. The authors also express a view on where corporate sustainability reporting is headed and conclude with a section on the future of sustainable reporting. This is a very important paper because it provides insights from two authorities looking to the future of corporate sustainability reporting and because a careful reading of the paper goes a long way towards an understanding of the differences between social and environmental accounting and sustainable accounting, for example:

However, it is essential to recognize that reporting about the social and the environmental - in whatever form it is undertaken- remains for us a distinctly different prospect from accounting (or reporting) for sustainability.

Sustainability is a systems concept and not an organizational concept. Sustainability is concerned with equity and justice, and with ecological limits as much as, if not more than, economic efficiency (Milne and Gray, UBOD 2007, p.195).

This is a 'must read' chapter!

Part III is entitled 'Accounting for sustainable development within organisations'; Chapter 11 is Full Cost Accounting (FCA) (Antheaume) which reviews the literature related to the inclusion of all costs, including externalities, in measuring economic activities. This may be achieved by either intervention in the market system (e.g. green taxes) or by the development of markets to permit trading (e.g. emissions trading). FCA is related to sustainability reporting, however, despite a thorough review of the literature the author concludes that more work is needed to better understand how the economic, social and environmental aspects of an entity interact with each other. This is the same conclusion arrived at by proponents of social and environmental accounting.

Bebbington (Chapter 12; Changing organizational attitudes and culture through sustainability accounting) examined the role that sustainability accounting disclosures may have in changing attitudes and culture within organizations. Change from social and environmental accounting to sustainable development (SD) is made more difficult by the nature of SD (refer the review of Milne and Gray above). The paper concludes that evaluating whether or not accounting interventions lead to change is difficult. Many organizations are change resistant. More in-depth and sustained empirical investigations are needed to shed light on this important area.

Ball and Grubric (Chapter 13 Sustainability accounting and accountability in the public sector) consider the relationship between sustainability accounting and public sector organizations which make up 40\% of GDP in many economies: 
...we see sustainability accounting and accountability as an opportunity to achieve a better accounting framework for PSOs in a post-NPM [New Public Management] world. By which we mean a framework in which community perspectives can help in accounting for the tasks of PSOs and PSO relationships and contributions in communities which live within the limits of ecological systems (UBOD 2007, p.260).

The authors suggest a number of avenues for further research in this area and conclude that they see sustainability disclosures as a key mechanism for engaging more people in the working of the public sector.

The final paper in Part III is Chapter 14 (Coulson; Environmental and social assessment in sustainable finance). Modern financing activities require an appreciation of social and environmental risks. Banks must be concerned with reputation within the banking community and this applies particularly to project finance. Socially responsible investment is also an important innovation. The author concludes that combining the needs of modern financing with sustainability accounting and reporting is a challenge:

A review of the contribution of sustainability accounting and reporting to finance decisions reveals that both borrowers and their financiers face problems establishing meaningful indicators for environmental and social performance and evaluating their impact on financial performance (UBOD 2007, p.278).

Part IV is devoted to other issues such as The nature of NGO accountability (O'Dwyer; Chapter 15). Non-Governmental Organizations (NGOs) are now subjected to increased scrutiny on a range of factors, including accountability and societal impacts. Aspects of NGO behavior and issues of accountability are considered in this chapter. NGOs come in many forms, some are concerned that pressures for accountability are greater on them than on either the private or government sectors. The author concludes that NGOs should not simply adopt accountability processes developed in the private sector, but should develop specific forms suited to the nature of NGOs:

The way a sector defines its accountability influences its distinctive identity and role in society. The NGO 'sector' addressed here is characterized by a distinctive accountability regime with different subjects and mechanisms of accountability. Consequently, these NGOs must develop their own tools, processes, and systems and then convince others of their efficacy and merit (UBOD 2007, p.299).

In Chapter 16 Dey considers Developing silent and shadow accounts, which may augment organisational accountability in a more satisfactory manner than might additional disclosures by management. The chapter reviews the history of Social Audit Ltd and Counter Information Services and defines their successors, silent and shadow accounts, reviews the literature to date, and provides a framework for thinking about silent and shadow accounts. Clearly there are conflicts between the academic approach which may measure published reports against published frameworks and those approaches which attempt to act as investigative journalism. Dey provides a framework aimed at the academic approach.

The contribution by Collison et al. (Chapter 17, Sustainability accounting and education) is timely. The chapter discusses the socialising effects of education, values implicit in accounting education and developments, challenges in SEA education, and education and the accounting profession. The perspectives of professional bodies are also considered. The literature is carefully reviewed, although there are two notable omissions; Lockhart and Mathews (2000) was 
in part a response to Cefcek et al. (1997) and Mangion (2005) has now appeared as a journal article. The importance of education in the development of more relevant accounting is summed up as follows:

Radicalized changes in humanity's outlook on sustainability will of course come - possibly in a matter of a few decades. The question is whether these changes will come quickly enough to avoid environmental and social catastrophe: for an enlightened and relatively gradual adaptation to take place, education - for accountants and many others - must be at least a necessary, if not a sufficient, condition (Collison et al., UBOD 2007, p.340).

The concluding chapter is the work of the three editors (Chapter 18; Postscript and conclusions). The authors argue that the various contributors have two distinct aspects requiring further elaboration. These are a focus on ecological space and carrying capacity of ecosystems, and a commitment to equity (on both an intra and inter-generational bases). It is the latter which presents the greatest challenge and produces the greatest opposition. The authors explain how current social and environmental disclosures fail to satisfy the needs of sustainable development:

In summary, the provision of an economic, social and environmental account of entity-level interactions falls short of constituting a full account of SD. In part the failure of accounting to achieve this end is due to structural impediments within society. If society cannot specify what constitutes social or ecological sustainability, any accounting system that draws its mandate from societal rules will also fall short of a SD account (Bebbington et al., UBOD 2007, p.347).

The overall sentiments of the various authors suggest collectively that the book marks a watershed in the development of non-traditional accounting, in which accounting for sustainability transcends social and environmental accounting developments.

\section{OBSERVATIONS ON TWO CONTRASTING WORKS}

As noted in the introduction, this paper reviews two contrasting books published at about the same time, publicized at the same conferences and aimed at similar audiences. GG 2007 (with the exception of the contribution by Milne) is firmly based in the 'traditional' social and environmental accounting literature, makes few if any references to sustainability, and has contributions from a number of well-known academics in the field who approach their subject from a number of philosophical positions. The material is sound and well-written, relatively uncontroversial, and constitutes the past and near-present (most papers would be two or more years old given the timetable for publication).

In contrast, UBOD 2007 is aimed at the future of the field and all the contributions are concerned at developing aspects of sustainable accounting. At a first reading the contributors to this tour de force appear to be marching into the future to the same SD/SA tune. However, a closer reading reveals that not all the contributors view SA in the same way. Some see it as social and environmental accounting with a more fashionable name, others go to considerable lengths to state what does and does not constitute SA, and a few argue that SA is an ideal state that probably does not or cannot exist. Similarly, it is suggested that to work on SA a critical theorist perspective is most useful, but some contributors do not appear to agree and espouse a social contract approach. So although the past/present versus future perspective appears to be useful in broadly categorizing the content of these books, UBOD 2007 is not as homogeneous in approach and philosophy as it appears to be at first sight. But the impression is created that one can be a social conservative and make a contribution to social and environmental accounting but 
to make a contribution to SA one must be more of a deep-seated radical position and probably follow a critical theorist paradigm. This would be unfortunate if it discouraged some researchers who could make important contributions to this debate.

There is perhaps a sense of déjà vu for this author since Mathews (1984) was an attempt to classify social accounting research in response to a literature that the author found confusing; and it appears that the development of SA is just as confused; perhaps there is an opportunity for a new researcher to attempt to unravel, understand and classify the different aspects of Sustainable Accounting!

\section{CONCLUDING COMMENTS}

This paper has reviewed two contrasting books, both authoritative in their respective fields and which together form a formidable resource for new or experienced researchers in social and environmental accounting and/or sustainable accounting. A close reading of both books should provide researchers whether beginners or more experienced, with a wealth of references, ideas, and inspiration. Whether wishing to pursue the more traditional social and environmental accounting or the more futuristic sustainability accounting research, there are so many opportunities that no academic no matter how experienced or inexperienced should lack for work to do. There will always be support, especially from those who contributed to both of these books.

There have been previous attempts to classify and categorize the social and environmental accounting, an opportunity now exists for a similar activity to be applied to sustainable accounting. Perhaps this idea might commend itself to an enthusiastic newcomer to the field!

\section{REFERENCES}

Bebbington, J. and Thomson, I. (2001). Commentary on : Some thoughts on social and wenvironmental accounting education, Accounting Education: An International Journal, 10(4) 353-355.

Gray, R.H. and Guthrie, J.E. (2007). Eds Social Accounting, Mega Accounting and Beyond: A festschrift in honour of M.R. Mathews. St Andrews:CSEAR.

Gambling, T.E. (1974). Societal Accounting, London: Allen and Unwin.

Lockhart, J.A. and Mathews, M.R. (2000). Teaching Environmental Accounting: A four-part framework, Advances in Accounting Education, 3, 57-84.

Mangion, D. (2005). An examination of social and environmental accounting in Australian Universities. Accounting Education: An International Journal, Supplement 1.

Mathews, M.R. (1984). A Suggested Classification for Social Accounting Research. Journal of Accounting and Public Policy, 3(3), 199-221.

Sefcik, S.E., Soderstrom, N.S. and Stinson, C.H. (1997). Accounting through green-colored glasses: Teaching environmental accounting, Issues in Accounting Education, 12(1) 129-140.

Unerman, J., Bebbington, J. and O’Dwyer, B. (2007). Eds Sustainability Accounting and Accountability. Oxford:Routledge. 